\title{
Una aproximación a la gestión cultural en el sitio arqueológico de Cajamarquilla, Lima- Perú
}

\section{An approach to cultural management in the archaeological site of Cajamarquilla, Lima- Peru}

\author{
Benito Cáceres Ramos \\ https://orcid.org/oooo-0002-9518-2032 \\ Ministerio de Cultura \\ bcra78@hotmail.com
}

\section{RESÚMEN}

En el presente artículo se hace la revisión de los informes finales de intervenciones arqueológicas, a partir de ello se aproxima a la gestión del patrimonio arqueológico en el sitio arqueológico de Cajamarquilla; los informes finales proporcionan información concerniente a la investigación, la conservación, la difusión y protección del monumento arqueológico. Asimismo, en relación a la difusión y protección se describe la función que cumple el Centro de Interpretación de Cajamarquilla implementada en el año 2010; lo antes descrito nos indica que estamos ante la presencia de la gestión del patrimonio cultural. En la parte final del texto, se hace algunas recomendaciones donde se considera importante la participación de los diferentes actores para proteger y gestionar el sitio arqueológico.

Palabras clave: reporte final; sitio arqueológico de cajamarquilla; gestión del patrimonio cultural.

\section{ABSTRACT}

In the present article, the final reports of archaeological interventions are reviewed, and from that review an approach to the archaeological heritage manage- 
ment in the archaeological site of Cajamarquilla was described. The final reports provide information concerning the investigation, conservation, diffusion and protection of the archaeological monument. Also, in relation to the diffusion and protection it describes the function that fulfills the Cajamarquilla's center of Interpretation implemented in the year 2010; the foregoing indicates that we are dealing with the presence of cultural heritage management. In the final part of the text, some recommendations are made where it is considered important the participation of the different actors to protect and manage the archaeological site.

Keywords: final reports; archaeological site of Cajamarquilla; cultural heritage management.

RECIBIDO: 19/07/2017 - ACEPTADO: 25/09/2020 - PubLICADO: 10/05/2021

\section{INTRODUCCIÓN}

El Perú es un país con gran diversidad de restos arqueológicos distribuidos a lo largo de su territorio, que son la expresión del desarrollo cultural alcanzado por la sociedad en cada región determinada, en donde se han adaptado al medio geográfico de la zona.

En la costa peruana una de esas manifestaciones culturales, es el Sitio Arqueológico de Cajamarquilla, ubicada en el valle medio de Lima, en la margen derecha del río Rímac.

Las investigaciones arqueológicas señalan ocupaciones que van desde la fase tardía del Intermedio temprano, leve presencia en el Horizonte Medio y en el Intermedio Tardío (Mogrovejo y Makowsky, 1999; Narváez, 2004).

En Cajamarquilla se han realizado proyectos de investigación arqueológica con diferentes objetivos, que han contribuido con nueva información, conservación, difusión y protección del sitio.

En el presente artículo se hace una aproximación a la gestión del patrimonio cultural en Cajamarquilla, a partir de la evaluación de los proyectos arqueológicos realizados en el sitio; observándose en sus inicios un predominio de proyectos con un carácter investigativo, incorporándose luego el componente de conservación y, finalmente, en los proyectos tardíos se incluyen la difusión y protección del monumento arqueológico.

En relación a la difusión y protección se describe la función que cumple el Centro de Interpretación de Cajamarquilla desde su implementación en el año 2010.

\section{UBICACIÓN}

El sitio arqueológico de Cajamarquilla se ubica en la localidad de Huachipa, en el distrito de Chosica, departamento de Lima. En la margen derecha del río Rímac, aproximadamente a 18 kilómetros de la ciudad de Lima, y los límites son los asen- 
tamientos humanos Casa Huerta la Campiña, Santa Cruz y el Paraíso por el Norte, el Ayllu y el cauce del río Huaycoloro por el Este, y campos de cultivo, viviendas y granjas por el Sur y Oeste (Franco, 1998). (Ver figura 01).

\section{DESCRIPCIÓN DEL SITIO ARQUEOLÓGICO}

El sitio arqueológico de Cajamarquilla ocupa una superficie extensa de aproximadamente 167 has. En relación a la cronología, se desarrolla en las fases tardías del Periodo Intermedio Temprano (500 d. C-650 d. C). Los edificios de estas épocas tienen similar organización interna en sus ambientes y estructuras que las pirámides Tello, Villar Córdova, Sestieri y Muelle (Mogrovejo, 1999). Asimismo, las investigaciones indican que Cajamarquilla fue reocupada en áreas específicas de las pirámides Tello y Sestieri, como cementerio ${ }^{1}$, durante el Periodo Horizonte Medio (500 d. C-1000 d. C.). Una nueva ocupación se desarrolla en Cajamarquilla durante el Período Intermedio Tardío (1000 d. C-1450 d. C), relacionado posiblemente al incremento del

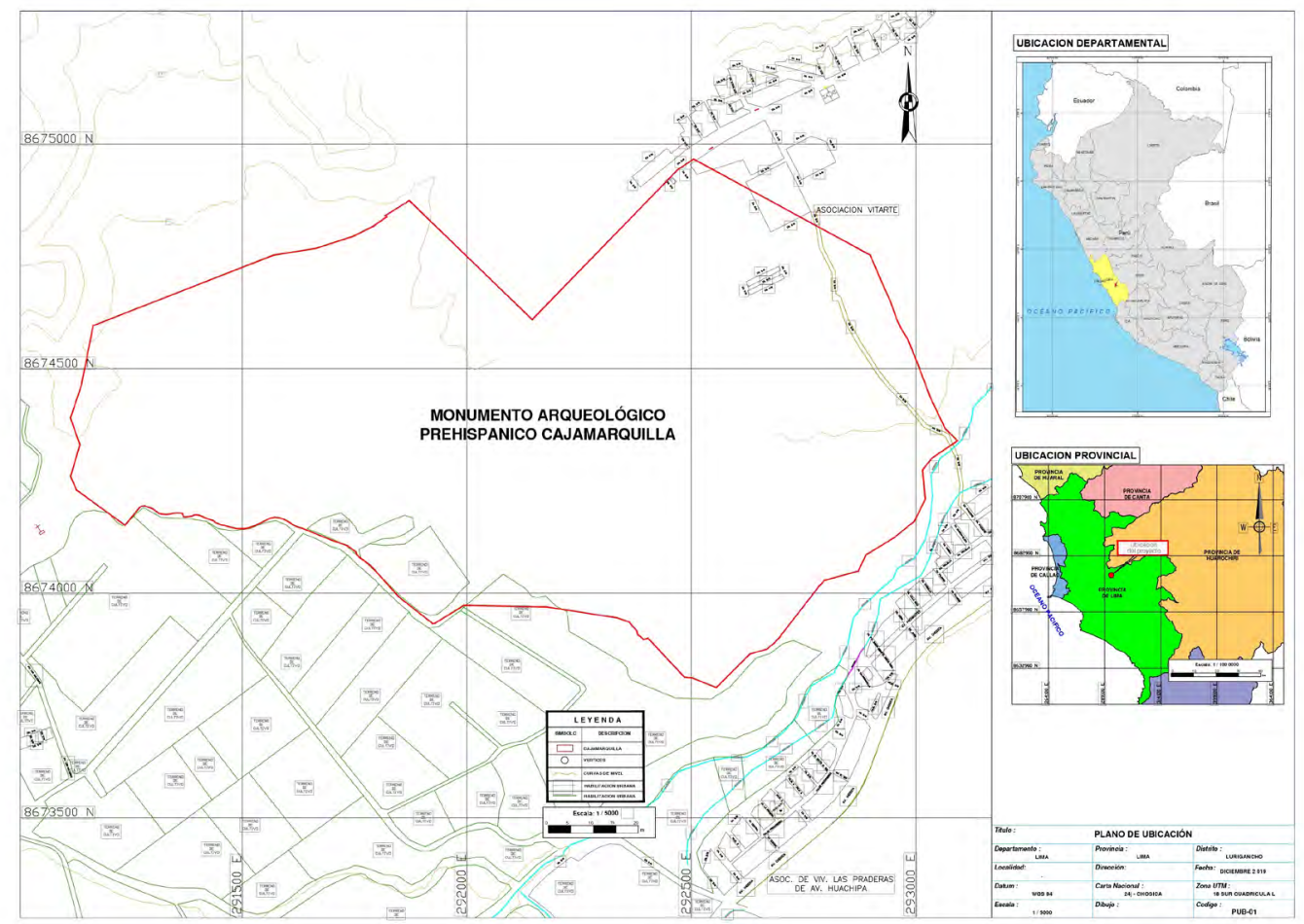

Figura 01. Plano de ubicación del Sitio Arqueológico de Cajamarquilla. Elaboración propia.

1. Los entierros extendidos corresponden a la época $1 \mathrm{~B}$ del Horizonte Medio y los enfardelados a las épocas 2A y 2B del Horizonte Medio, en el primer caso cuando las pirámides estaban abandonadas y en el segundo caso las pirámides estaban abandonadas (Narváez, 2006). 
caudal del río Huaycoloro que se manifiesta en las construcciones de arquitectura monumental en esta época (Mogrovejo y Makowski, 1999).

Cajamarquilla tiene características de una ciudad prehispánica presenta edificios, recintos, plazas, patios, áreas de depósitos, calles, accesos, banquetas, muros (tamaño variable de tapia, algunos en pie y otros colapsados), con diferentes técnicas de construcción; sobresaliendo por su volumen y denominadas con los nombres de los investigadores: Conjunto Villar Córdova, Conjunto Sestieri, Conjunto Jorge C. Muelle y Conjunto Tello, Conjunto Kroeber y D'Harcourt (Franco, 1998).

Una de las descripciones más aceptadas por los investigadores para Cajamarquilla es el realizado por el arqueólogo Alberto Bueno en 1974-1975, quien los clasifica en los siguientes tipos de sectores:

1) Grupos con grandes pirámides rodeados con recintos y plazas, que constituyen cuatro grandes grupos o conjuntos arquitectónicos y que recibieron los nombres de algunos de los arqueólogos que trabajaron en el sitio: "Conjunto Villar Córdova" al noroeste, "Conjunto Sestieri" y "Conjunto Jorge C. Muelle" al centro, y "Conjunto Tello" al este.

2) Grupos amurallados con una pequeña pirámide dominante, como los conjuntos "Kroeber" y "D'Harcourt"

3) Grupos amurallados sin pirámide como el "Conjunto Laberinto"

4) Áreas de servicio doméstico. (Narváez, 2004, p.14). (Ver figura 02)

\section{GESTIÓN DEL PATRIMONIO CULTURAL}

En el presente estudio se considera el siguiente marco teórico sobre gestión del patrimonio cultural.

\section{Patrimonio cultural}

Una de las más aceptadas definiciones del término patrimonio cultural, es la dada por la Organización para la Educación, Ciencia y la Cultura de las Naciones Unidas en la Convención sobre la Protección del Patrimonio Mundial Cultural y Natural de París (UNESCO, 1972), que señala:

Artículo $1^{\circ}$.- A los efectos de la presente convención se considerará "patrimonio cultural", los monumentos: obras arquitectónicas, de escultura o de pinturas monumentales, elementos o estructuras de carácter arqueológico, inscripciones, cavernas y de grupos de elementos que tengan un valor universal excepcional desde el punto de vista de la historia, del arte o de la ciencia.

Los conjuntos: grupos de construcciones, aisladas o reunidas, cuya arquitectura, unidad e integración en el paisaje les dé un valor universal excepcional desde el punto de vista de la historia, del arte o de la ciencia. 


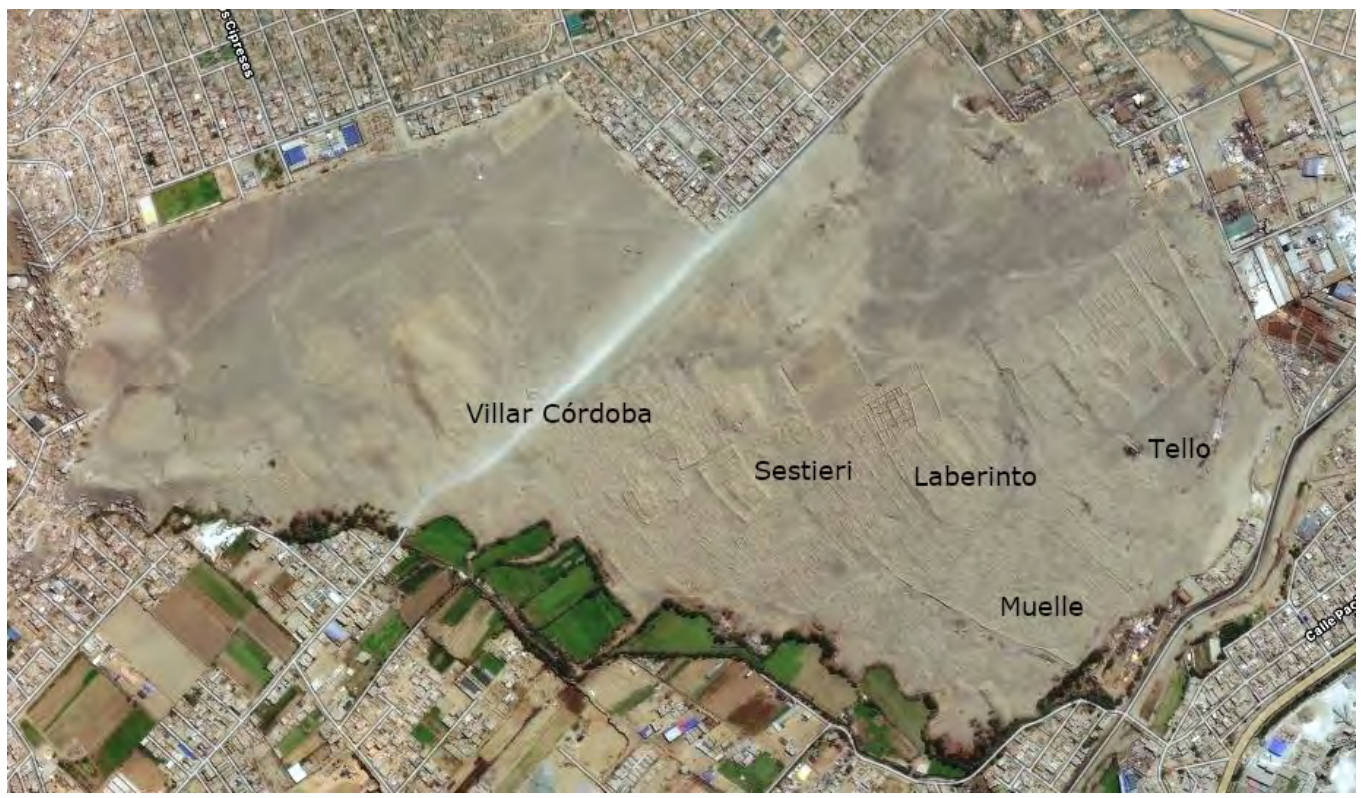

Figura 02. (Imagen satelital) Tomada de: https://satellites.pro/mapa_de_Huachipa. Lima_region.Peru\#-11.985015,-76.908889,16. Revisada el 18/03/2018. Se trabajó sobre la imagen satelital para ubicar los conjuntos de Cajamarquilla.

Los lugares: obras del hombre u obras conjuntas del hombre y la naturaleza así como las zonas, incluidos los lugares arqueológicos que tengan un valor universal excepcional desde el punto de vista histórico, estético, etnológico y antropológico.

De la misma forma, en la Declaración Universal sobre la Diversidad Cultural de la (UNESCO, 2001) en el Artículo $7^{\circ}$ define este término:

El patrimonio cultural, fuente de la creatividad. Toda creación tiene sus orígenes en las tradiciones culturales pero se desarrolla plenamente en contacto con otras. Esta es la razón por la cual el patrimonio, en todas sus formas, debe ser preservado, valorizado y transmitido a las generaciones futuras como testimonio de la experiencia y de las aspiraciones humanas, a fin de nutrir la creatividad en toda su diversidad e instaurar un verdadero diálogo entre las culturas.

El Ministerio de Cultura del Perú (2015), sobre el patrimonio cultural, precisa que:

Es la herencia de bienes materiales e inmateriales que nuestros padres y antepasados nos han dejado a lo largo de la historia. Se trata de bienes que nos ayudan a forjar una identidad como nación y que nos permiten saber quiénes somos y de dónde venimos, logrando así un mejor desarrollo como personas dentro de la sociedad. 
Todas las personas formamos parte de una familia pero somos, al mismo tiempo, integrantes de una comunidad, de una región, de un país. De la misma manera en que heredamos bienes materiales y tradiciones familiares, recibimos también el legado de la cultura que caracteriza a la sociedad donde crecemos y nos desarrollamos. Estas expresiones distintivas que tenemos en común como la lengua, la religión, las costumbres, los valores, la creatividad, la historia, la danza o la música son manifestaciones culturales que nos permiten identificarnos entre nosotros y sentir que somos parte de una comunidad determinada y no de otra. Esta herencia colectiva es el patrimonio cultural.

\section{Patrimonio arqueológico}

Al respecto, se considera lo expuesto, en la Carta Internacional para la Gestión del Patrimonio Arqueológico ${ }^{2}$, que señala:

El "patrimonio arqueológico" representa la parte de nuestro patrimonio material para la cual los métodos de la arqueología nos proporcionan la información básica. Engloba todas las huellas de la existencia del hombre y se refiere a los lugares donde se ha practicado cualquier tipo de actividad humana, a las estructuras y los vestigios abandonados de cualquier índole, tanto en la superficie, como enterrados, o bajo las aguas, así como al material relacionado con los mismos.

Siguiendo esta línea de conceptualización el Convenio Europeo sobre la Protección del Patrimonio Arqueológico (Revisado), aprobado en La Valetta (1992) precisa:

“A este fin se considerarán elementos del patrimonio arqueológico todos los restos y objetos y cualquier otra huella dejada por la humanidad en épocas pasadas". Asimismo, precisa "el patrimonio arqueológico incluye estructuras, construcciones, grupos de edificios, obras de ingeniería civil, objetos transportables y monumentos de cualquier otro tipo, así como su contexto, sea sobre tierra o bajo el agua".

En ese sentido, los sitios arqueológicos: "Son espacios con evidencia de actividad humana realizada en el pasado, con la presencia de elementos arquitectónicos o bienes muebles asociados de carácter arqueológico, tanto en superficie como en el subsuelo"3. Es decir, el sitio de Cajamarquilla, de acuerdo con lo descrito líneas arriba es patrimonio arqueológico, por ende, es parte del patrimonio cultural.

\section{Gestión del patrimonio cultural}

¿Qué es gestión del patrimonio cultural?. La Asociación Española de Gestores del Patrimonio Cultural, la define como: "la eficiente administración de recursos (cultu-

2. Preparada por el Comité Internacional para la Gestión del Patrimonio Arqueológico (ICAHM) y adoptada por la Asamblea General del ICOMOS en Lausana, Suiza, (1990)

3. Reglamento de Intervenciones Arqueológicos, (2014). Decreto Supremo № 003-2014-MC. Pg. 16 
rales, humanos, económicos y de todo tipo) ordenada a la consecución de objetivos sociales que afecten al patrimonio cultural" (Zamora, 2002, p. 8).

La gestión del patrimonio cultural no solo consiste en identificar, proteger y presentar al público las expresiones de la cultura material e inmaterial de los pueblos en un contexto de cambio permanente, sino que implica, a su vez, un proceso de selección, jerarquización y control de las diversas manifestaciones culturales en clasificaciones que suelen delimitar el carácter de tal gestión (Mariano, Endere y Mariano, 2014, p. 244).

Por su parte, Monsalve (2011), señala:

La gestión del patrimonio cultural se vierte en un proceso participativo construido en la cotidianeidad y de forma conjunta y abierta, a través de la interacción de diferentes actores que cooperan entre sí para disfrutar de ese patrimonio, y asegurar al mismo tiempo su transmisión a las generaciones futuras y su viabilidad en el tiempo. (p. 39).

En el Libro Blanco de la gestión del Patrimonio Histórico-arqueológico del Grupo de Ciudades Patrimonio de la Humanidad de España/UNESCO. Ministerio de Educación, Cultura y Deporte (2015), se indica:

La gestión del patrimonio cultural es un proceso ligado a un concepto fundamental que es la cadena de valor de la producción del patrimonio cultural. El patrimonio cultural es una categoría social, en puridad no existe realmente, sino que se crea y se le debe dotar de contenido o, dicho de otro modo, de sentido. Como todo proceso de gestión debe estar bien protocolizado pero también debe ser suficientemente flexible para adaptarse a los cambios del entorno. La investigación, la protección, la conservación, la interpretación, la socialización y la evaluación del impacto del patrimonio cultural son los cimientos de un edificio que puede quedar inconcluso $\mathrm{y}$, por lo tanto, en peligro si falta cualquiera de ellos. (p. 29).

Respecto a la conservación, Zúñiga y Pérez (2013), sostienen que, “contempla el conjunto de acciones regulatorias encaminadas a preservar o enriquecer el valor patrimonial; incluye tanto la utilización como el conjunto de acciones dirigidas a aprovechar el valor patrimonial, de acuerdo con el contexto social y económico". (p. 77). Indicando también que debe considerar acciones de restauración, conservación, preservación y protección.

En relación a la interpretación, Hernández (2002), sostiene:

La presentación ("interpretación" o "puesta en valor" según es denominada por distintos autores) debe incorporar todos estos aspectos a la hora de administrar y dar acceso a este patrimonio al resto de la humanidad, (...). La presentación puede darse bajo la forma de museos, centros de interpretación, parques 
culturales y/o naturales, "circuitos culturales y/o naturales", con sendas y postas específicas, sitios arqueológicos o históricos administrados, etc. (p. 132).

\section{ANTECEDENTES}

\subsection{Investigaciones iniciales}

De acuerdo a Bueno (1974-75), uno de los primeros documentos que menciona a Cajamarquilla son:

Los informes de Toledo en la parte que toca a las reducciones de indios en "Los Reyes". Mr. de Laporte en su libro "El viajero Universal" de 1799 la llamó "Caxamarca la Vieja" ubicándola en el "valle que llaman Guachipa al nordeste de Lima". (Narváez, 2004, p. 16).

\subsection{Viajeros}

Las referencias más detalladas con descripciones, dibujos e hipótesis de trabajo se realizan en la segunda mitad del S. XIX.

En el año de 1864, Ephrain George Squier exploró Cajamarquilla, así como otros sitios arqueológicos del país. Fue el primer investigador que efectuó un estudio sobre este sitio arqueológico realizando descripciones, dibujos y un plano de los sectores Laberinto y Muelle, (...).

Ernst W. Middendorf a fines del S. XIX señalaba que Cajamarquilla pudo haber albergado de diez mil a doce mil habitantes, presentando estructuras formadas por muros de adobones muchas de las cuales se habían desplomado, según él por obra de terremotos. Refiriéndose a las grandes pirámides dijo que debieron ser fortalezas y acerca de los pozos que distingue por todas partes, afirmó que algunos pudieron ser tumbas, aunque las que se ubicaban en pequeñas plazas pudieron servir de graneros o depósitos de víveres. (Narváez, 2004, p. 16).

\subsection{Investigadores en el siglo XX}

Durante este siglo se realizaron las primeras investigaciones arqueológicas de carácter científico con la finalidad de ver la historia cultural de Cajamarquilla.

En 1906 Max Uhle recorrió el sitio y excavó en un cementerio ubicado cerca del límite noroeste del núcleo con arquitectura intensiva en terrenos de la hacienda Nievería (...), el análisis posterior del material obtenido por Uhle ha develado que el cementerio estuvo en uso desde fines del periodo intermedio temprano hasta la época 2A del Horizonte Medio. ${ }^{4}$

En 1935, Jorge C. Muelle realiza excavaciones en otro entierro parcialmente alterado muy cerca de los trabajos realizados por Uhle, debido a las características y la 
cercanía del cementerio de Nievería, ha servido para fechar por extensión el sitio arqueológico de Cajamarquilla (Segura, 2001).

El monseñor Pedro Villar Córdova (1935) escribe sobre Cajamarquilla en un gran intento por descifrar la historia del complejo. Asimismo, entre los años 1938- 1939, el Dr. Alberto Giesecke (1939), a la par de sus excavaciones en Pachacamac realiza limpieza y refacciones en el Grupo Tello (Franco, 1998).

En 1944, J.C. Tello, realizó excavaciones en el sitio (Ravines 1988:28); sus informes se encuentran depositados en el "archivo Tello" en la Casona de la Universidad Nacional Mayor de San Marcos y no han sido publicados. (Narváez, 2006, p. 28).

A partir de 1962 la Misión Arqueológica Italiana realiza un programa de investigaciones a largo plazo que se extendió hasta 1970, auspiciada por el gobierno de ese país. Por la extensión de los trabajos realizados y por la cantidad de material recuperado esta es una de las intervenciones más importantes desarrolladas en el sitio, (...) (Segura, 2001). Las excavaciones de la Misión Arqueológica Italiana en el Conjunto Julio. C. Tello "dieron como resultado, una serie de contextos funerarios fechados estilísticamente en el Horizonte Medio $1 \mathrm{~B}$ y $2 \mathrm{~A}^{5}$, (...). Tales contextos muestran una eminente correspondencia con el cementerio excavado por Uhle." (Segura, 2001, p. 30).

Se reinician actividades arqueológicas en el Conjunto Tello siendo excavada en 1982 por el arqueólogo Odón Rosales, cuyos resultados no han sido publicados. En 1985 el Dr. Arturo Jiménez Borja, realizó labores de reconstrucción y protección del sitio, especialmente en el sector llamado El Laberinto. Para tales fines se practicaron algunas excavaciones de extensiones limitadas. (Walde, 2006-2007, p. 6).

Un nuevo programa sostenido de investigaciones se desarrolla en el sitio, desde 1996 hasta 2007, el Proyecto Arqueológico Cajamarquilla.

Durante los años 1996 y 1997 los trabajos se realizan bajo la dirección del arqueólogo Juan Mogrovejo en el conjunto arquitectónico Julio C. Tello (...). Posteriormente, en 1998 y 1999 el arqueólogo Dante Casareto realiza investigaciones en el Conjunto Tello y Conjunto Muelle. En el año 2000 y 2001 Rafael Segura, continúo con las investigaciones en el Conjunto Villar Córdova de Cajamarquilla. (Walde, 2006-2007, p. 6).

Entre los años 2002 y 2003, la dirección del proyecto estuvo a cargo del arqueólogo Héctor Walde, quien realizó limpieza y excavaciones en el Conjunto Sestieri. En el año 2004 y 2005 las investigaciones estuvieron a cargo de Iván Ghezzi, realiza el levantamiento planimétrico de los Conjuntos Tello y Sestieri (Ghezzi, 2007). Asimismo, en el año 2004, se realizaron dos intervenciones que contribuyeron a la conser-

4. El Horizonte Medio, es el periodo que comprende aproximadamente entre los 800 y 1100 años D.C. Menzel, 1968, p, 14.

5. Corresponde a una de las 4 épocas en que se divide el Horizonte Medio: 1A, 1B, 2A, 2B, 3 y 4. Menzel, 1968, p. 14. 
vación de las estructuras del sitio: 1 . Intervenciones de emergencia en la calle E-0; y 2: Intervenciones pilotos en la Huaca Sestieri (Walde, 2006-2007).

Entre los años 2006 y 2007, el proyecto estuvo a cargo del arqueólogo Héctor Walde, quien desarrollo el programa de conservación en las estructuras de barro del Conjunto Tello, análisis de materiales recuperados en las temporadas 2002 al 2004, y realizó trabajos de conservación del sitio a través de programas sociales con la comunidad. (Walde, 2006-2007, p. 4).

\section{INTERVENCIONES DE CONSERVACIÓN}

Como se ha mencionado en los antecedentes del presente texto, los proyectos arqueológicos en Cajamarquilla se desarrollan con el fin de investigar y obtener nueva información y conocimientos sobre el sitio. En algunos de estos proyectos también han contemplado realizar la conservación de estructuras de barro debido al deterioro en que se encontraban; así como en los materiales arqueológicos recuperados en las excavaciones (cerámica, óseo, textil, metal, etc.), en Cajamarquilla.

Uno de los primeros trabajos de conservación y restauración se realizó en 1985, por el Dr. Arturo Jiménez Borja, quien efectúa una serie de intervenciones de reconstrucción y protección del sitio, en el sector denominado, El Sector Laberinto. (ver figura 03).

Asimismo, en los diferentes proyectos arqueológicos en Cajamarquilla se han realizado trabajos de conservación en las estructuras de barro y en los materiales arqueológicos, a continuación detallamos:

- Informe Final del Proyecto Arqueológico Cajamarquilla temporada 1997, señala: "En campo hemos consolidado muros en grave peligro de colapso, hemos cubierto recintos enteros para su mejor protección, hemos sellado accesos utilizados por los pobladores de los asentamientos humanos vecinos y por los pastores de cabras" (Mogrovejo, 1997, cuarto párrafo de la introducción).

Asimismo en relación a los materiales arqueológicos indica:

(...), en el análisis de material han avanzado en general hasta en un $80 \%$ con lo obtenido en la campaña 1996, y en un 10\% con lo obtenido en la campaña 1997. En cuanto a la conservación hemos logrado la recuperación de casi el $90 \%$ de nuestro material funerario, con más de 50 objetos adecuadamente restaurados; habiéndose almacenado el material restante de excavación en condiciones adecuadas y con todas las medidas necesarias para su protección y conservación" (Mogrovejo, 1997, tercer párrafo de la introducción).

- Informe Final del Proyecto Arqueológico Cajamarquilla temporada 1999, se menciona: "se ha consolidado los muros muy erosionados, haciendo una diferencia de 


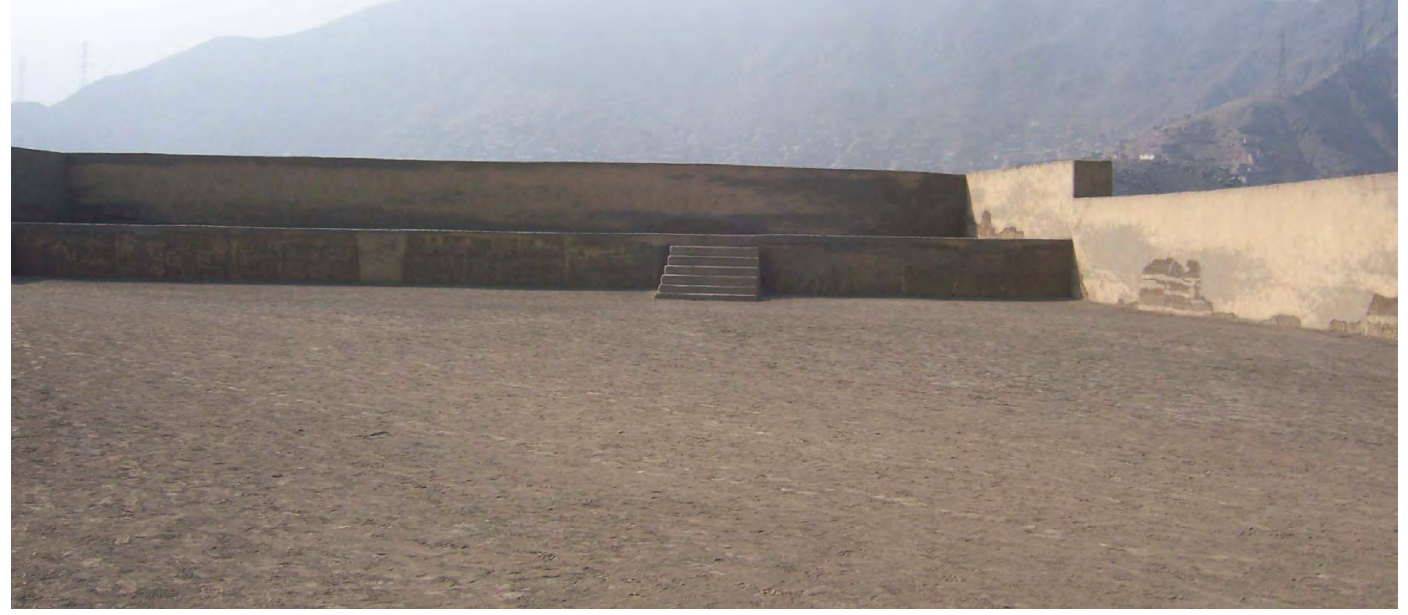

Figura 03: detalle del Sector Laberinto. Registro personal tomado en el 2013.

la parte original, además se realizó anastilosis en la Plaza Norte”, (...)”. En relación a los materiales de excavación señala: "se han realizado trabajos de conservación, en material cerámico y textiles, los cuales fueron embalados en cajas de cartón siguiendo un orden: grupo, sector, unidad, capa, nivel, material logrando disminuir el riesgo del material arqueológico". (Casareto, 2000, p. 216).

- Informe final del Proyecto Arqueológico Cajamarquilla en el año 2003, menciona: "Se ha considerado que la conservación de los objetos y estructuras excavadas como responsabilidad y parte integral de este proyecto arqueológico" (Walde, 2003, p. 24).

- Informe Final del Proyecto Arqueológico Cajamarquilla del 2004 y 2005, señala: “(...), intervenciones de emergencia en la Calle Inca y una intervención piloto en el sector Sestieri" (Ghezzi, 2007). Asimismo, el informe contiene fichas de intervención de estructuras que señalan se intervino mediante la conservación la Calle Inca y el Complejo Administrativo Ceremonial (Conjunto Sestieri): Ver figuras 04 y 05.

Sobre el trabajo de los materiales recuperados señala: "en la temporada 2004-5 hemos trabajado con la colección textil de las temporadas 2000 y 2003, procedentes de los sectores Villar Córdova y Sestieri respectivamente" (Ghezzi, 2007). En el cual se han realizado un estudio estilístico, conservación preventiva, registro e inventario y almacenaje de los textiles. 


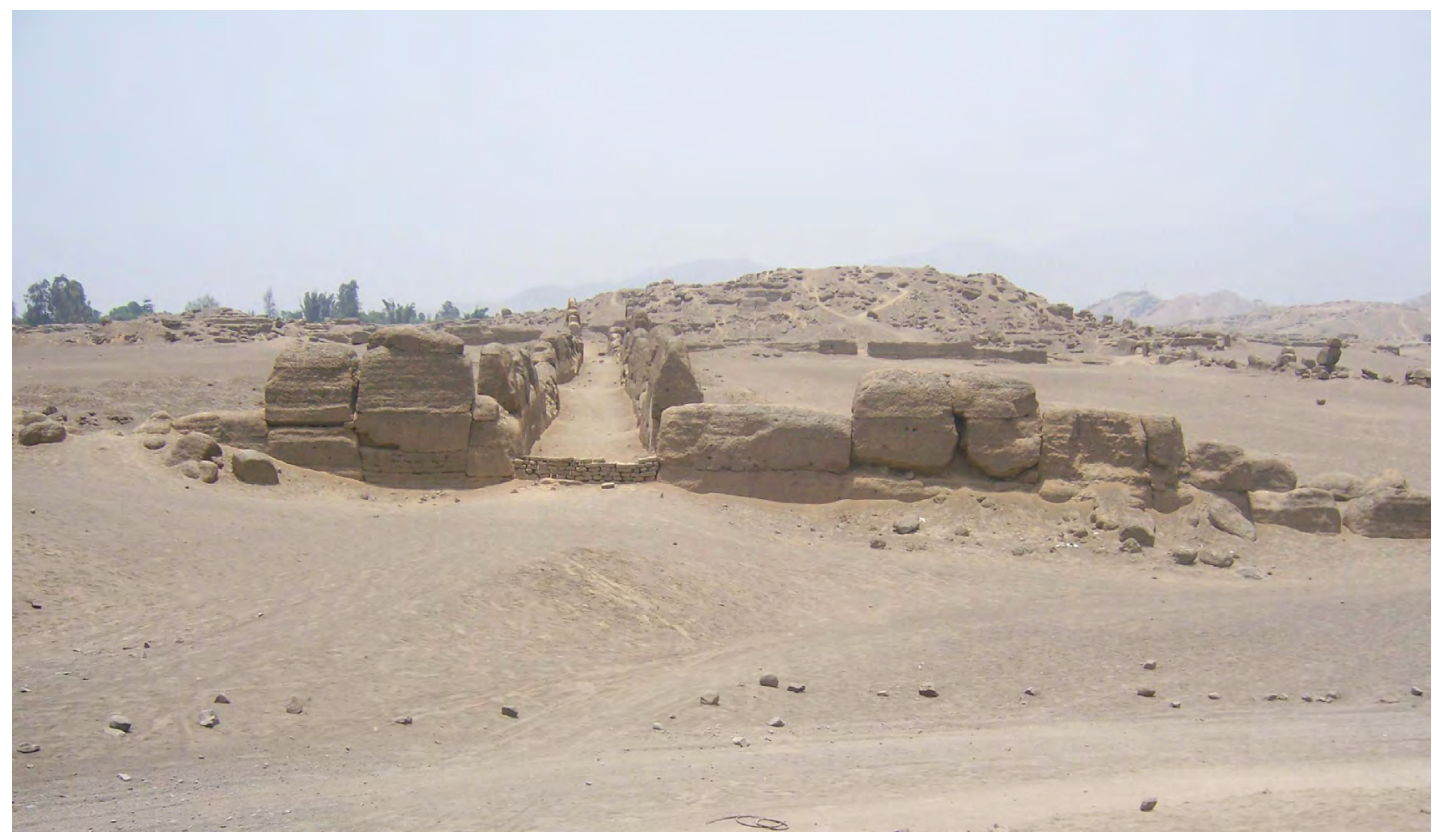

Figura 04: detalle de la Calle Inca, al fondo se observa el Conjunto Sestieri. Registro personal tomado en el 2013.

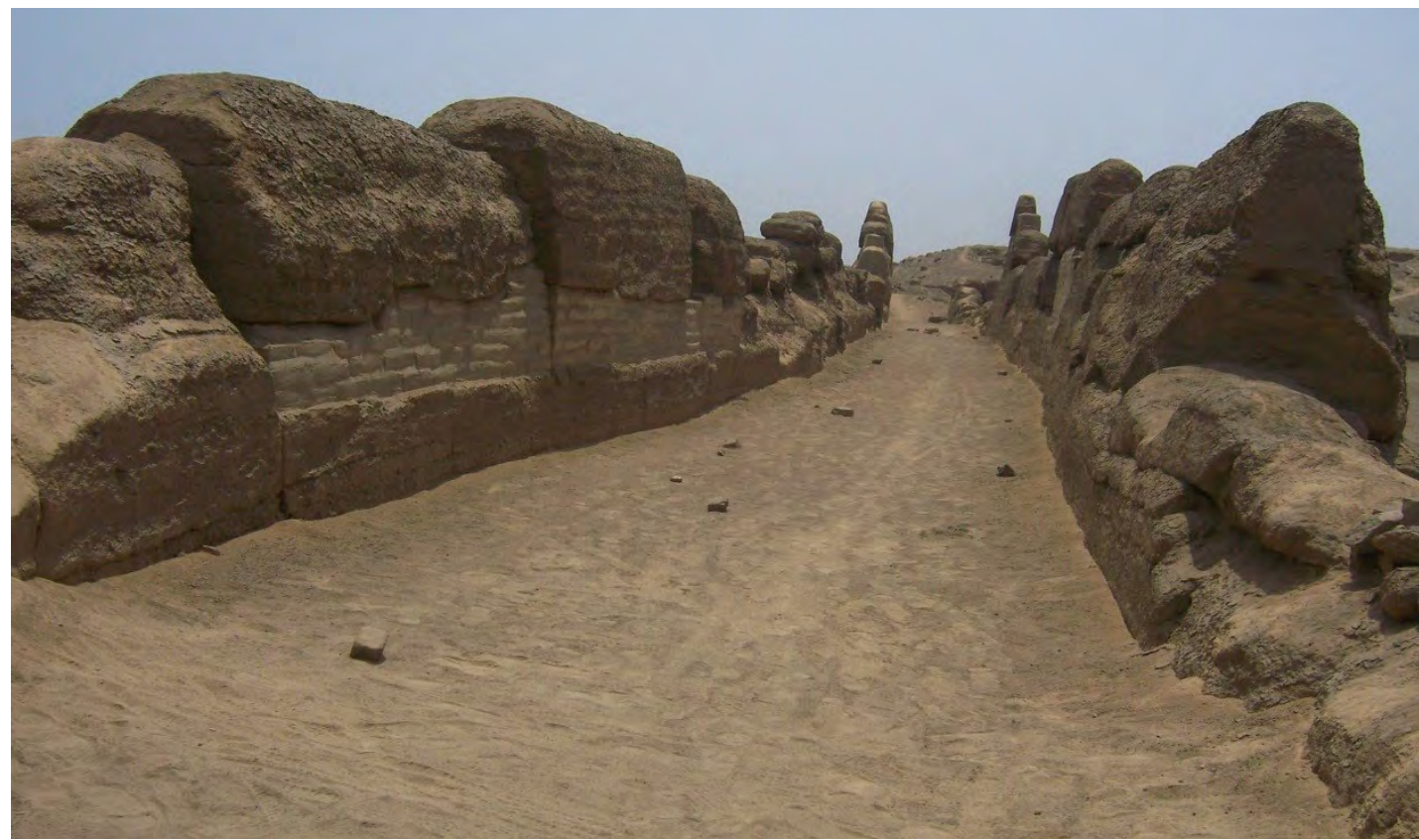

Figura 05: detalle de la Calle Inca, se observa los trabajos de conservación realizados 
- Informe Final del Proyecto Arqueológico Cajamarquilla del 2006 y 2007, indica: “(...), se realizó una evaluación detallada del estado de conservación de las estructura del Conjunto Tello, junto con la investigación de los materiales que la componen con la finalidad de establecer el procedimiento de conservación más adecuado para las estructuras". (Walde, 2006-2007, p. 12).

\section{PROTECCIÓN Y DIFUSIÓN DEL SITIO ARQUEOLÓGICO}

A continuación se hace referencia a los informes finales de proyectos arqueológicos en Cajamarquilla, que incluyen el componente de protección y difusión:

- Informe Final del Proyecto Arqueológico Cajamarquilla temporada 1997:

Finalmente en cuanto a la difusión de los resultados de nuestros proyectos, son más de 10 los reportajes periodísticos en los cuales no solo mencionamos los datos científicos que se obtienen, sino también enfatizamos la importancia del cuidado y conservación de Cajamarquilla. Con el apoyo de la Sala de la Cultura del Banco Wiese hemos logrado presentar una muestra durante los meses de setiembre y octubre con más de 3, 500 visitantes, de los cuales 600 corresponde a estudiantes y profesores de Cajamarquilla. Hemos efectuado también charlas con los pobladores, y hemos coordinado con los dirigentes para la prevención de incidentes que alteren el actual statu quo en cuanto a las ruinas; todo lo cual creemos ha sido positivo para lograr en los pobladores de los asentamientos humanos vecinos una nueva visión respecto a las ruinas, óptica en el cual está presente el respeto por el patrimonio y las instancias gubernamentales que están a cargo de la protección del lugar. (Mogrovejo, 1997, quinto párrafo de la introducción).

- Informe Final del Proyecto Arqueológico Cajamarquilla temporada 1998:

(...), en torno a la protección del lugar, se coordinó con la Dirección de Sitios Arqueológicos del I.N.C. y los pobladores de los asentamientos humanos El Paraíso, Santa Cruz de Jicamarca, Casa Huerta La Campiña, Cooperativa el Ayllu, agricultores y criadores de chanchos clandestinos, quienes afectan de una u otra manera el área intangible en la colocación de hitos de concreto, a fin de tener una frontera fija. (Casareto, 1999).

- Informe Final del Proyecto Arqueológico Cajamarquilla temporada 1999:

(...), la presencia del equipo en la arqueológica de Cajamarquilla, permite las coordinaciones con los diferentes grupos poblacionales, brindando apoyo a la seguridad de la zona, y motivando a la población el respeto, al trabajo realizado por nosotros. La presencia del Instituto Nacional de Cultura, es determinante en la protección del sitio, sin embargo es necesario continuar con las 
campañas de difusión de los trabajos realizados..., hemos repintado dos letreros de señalización dentro del complejo arqueológico. (Casareto, 2000, p. 223).

- Informe Final del Proyecto Arqueológico Cajamarquilla del 2003:

Sexto programa de difusión del Proyecto Arqueológico Cajamarquilla (PACYW), se ha elaborado diferentes programas que involucran y benefician directamente a la población local, y que paralelamente son centro de expedientación que apoyan los requerimientos de la investigación científica y puesta en valor del sitio arqueológico. Estos programas están de alguna manera relacionados con las actividades con lo cual esta población se puede identificar, como por ejemplo, la actividad artesanal, la creación de áreas verdes, producción agrícola, etc. (Walde, 2003, p. 25).

Asimismo, el citado informe final en el Noveno programa, Educación Ciudadana, señala:

Este programa está dirigido tanto a la población infantil local como a adultos involucrados en la tarea de educar, (...); tiene como principal objetivo concientizar sobre la importancia de la preservación del Patrimonio Cultural en general y en especial difundir información sobre la importancia del sitio de Cajamarquilla en el pasado y sus posibilidades en el presente y su potencialidad para el futuro. (Walde, 2003, p. 27).

- Informe Final del Proyecto Arqueológico Cajamarquilla del 2006 y 2007, señala:

Se ha desarrollado el Programa de difusión del Sitio Arqueológico de Cajamarquilla, cuyo objetivo principal es dar a conocer el Sitio Arqueológico de Cajamarquilla al público en general. Una serie de visitas guiadas han sido realizadas a lo largo de esta temporada; se han presentado los avances de los trabajos efectuados en los diversos programas en los siguientes eventos:

- Conferencia Ciencia y desarrollo: la reconstrucción de técnicas prehispánicas. En mayo del 2006.

- Presentacion del The Ancient City of Cajamarquilla: Use of the Archaeological site for the benefit of the community, en the 34th Annual Meeting of the American Institute for conservation of historic and Artistic Work (AIC) Providence, Rhode Island, EEUU. Junio del 2006.

- En los meses de enero y febrero del 2007, el Proyecto Arqueológico Cajamarquilla-Yachay Wasi (PAC-YW), participo de la muestra itinerante de la cultura peruana a bordo de la Armada peruana "Mollendo" visito los puertos de Valparaiso, San Diego, Balboa, Acapulco y Quito.

- Como parte del programa dirigido a las Instituciones Educativas de zona de Cajamarquilla se ha elaborado una Guía Metodológica para docentes del cuarto año de primaria. 
- Se han realizado otras actividades de difusión como el uso de la página Web y publicaciones. (Walde, 2006-2007, p. 23).

\section{EL CENTRO DE INTERPRETACIÓN DE CAJAMARQUILLA (CIC)}

En el año 2010 se inauguró el Centro de Interpretación de Cajamarquilla, con el financiamiento y la participación de la ONG Cesal, el INC, La Universidad Privada Cede Sapientae, y La Agencia Española para la Cooperación Extranjera y actualmente está bajo la administración del Ministerio de Cultura.

De acuerdo al organigrama del Ministerio de Cultura, el Centro de Interpretación de Cajamarquilla, es un Módulo descentralizado de Participación Ciudadana, dependiente de la Dirección de Participación Ciudadana que pertenece Dirección General de Defensa del Patrimonio Cultural.

El Centro de Interpretación de Cajamarquilla se creó con la finalidad de implementar un espacio participativo para la defensa, protección y preservación del patrimonio cultural de la Zona Arqueológica Monumental de Cajamarquilla (figura 6).

\section{Logros}

- Disminución de las afectaciones tanto en cantidad como en gravedad.

- La población cuenta con herramientas, espacios y oportunidades para participar en la defensa, protección y conservación del patrimonio cultural local.

- Las instituciones educativas cuentan con herramientas e información necesaria para la integración del patrimonio cultural y la historia local dentro de la currícula educativa.

- La población gasta menos tiempo en realizar consultas gracias a la implementación del CIC.

- El patrimonio cultural contribuye al desarrollo social de la localidad ${ }^{6}$

\section{ESTADO DE LA CUESTIÓN}

La gestión del patrimonio cultural, considera que la investigación, la protección, la conservación, la interpretación, la socialización y la evaluación del impacto del patrimonio cultural son los cimientos de un edificio que puede quedar inconcluso $y$, por lo tanto, en peligro si falta cualquiera de esos pasos ${ }^{7}$. La investigación es fundamental para el desarrollo del conocimiento sobre una determinada materia. Los proyectos en Cajamarquilla han sido planteadas desde esta perspectiva, como las efectuadas por Max Uhle (1906), Jorge C. Muelle (1935), Alberto Giesecke (1939), Julio C. Tello (1944), La Misión Arqueológica Italiana (1962 -1970), Odón Rosales (1982), Arturo Jiménez (1985), Proyecto Arqueológico Cajamarquilla (PAC) (1996-2007): bajo 


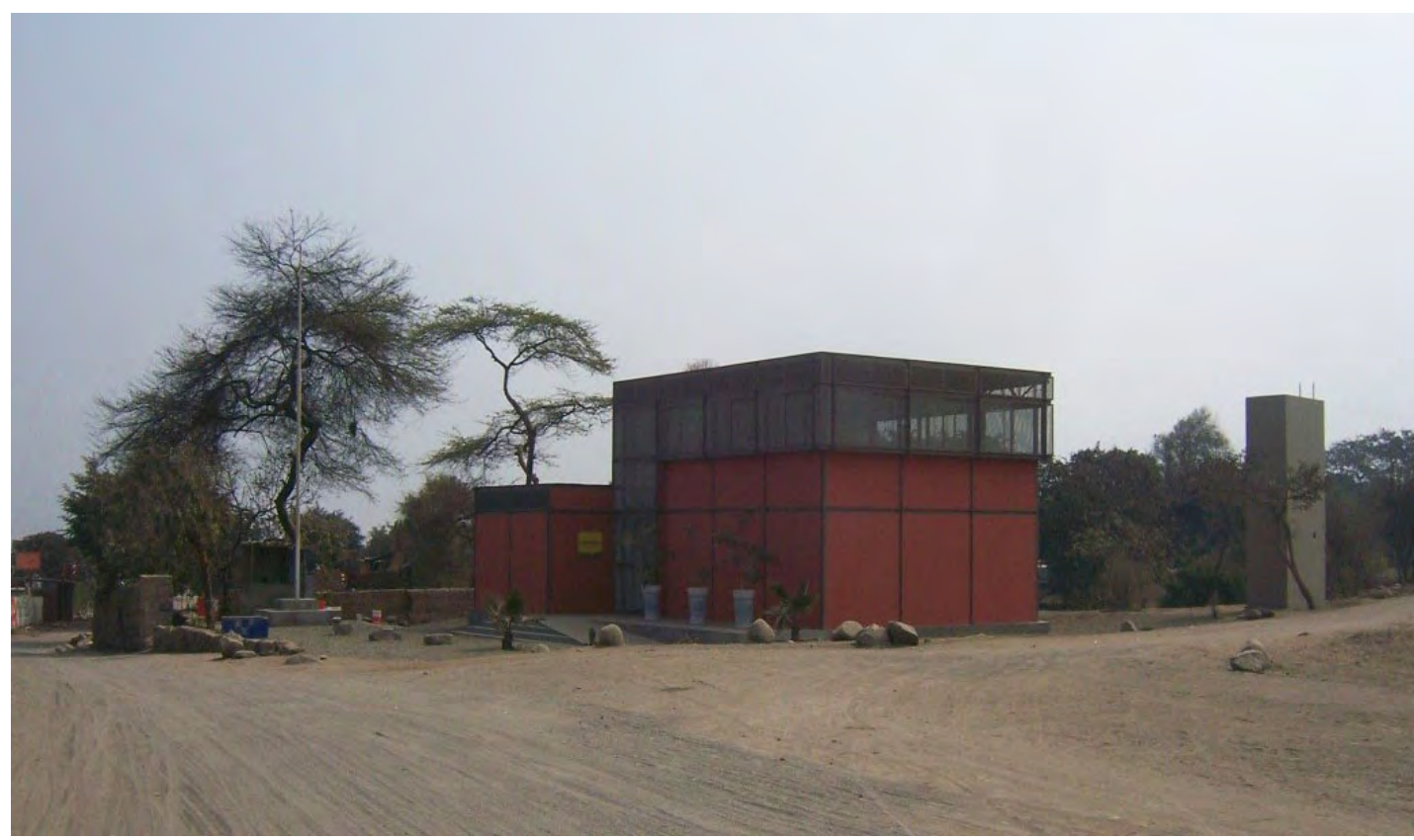

Figura 6: Centro de Interpretación de Cajamarquilla. Registro personal tomado en el año 2011.

la dirección de diferentes arqueólogos: Juan Mogrovejo (1996-1997), Dante Casareto (1998-1999), Rafael Segura (2000-2001), Héctor Walde (2002 y 2003), Iván Ghezzi (2004-2005) y Héctor Walde (2006 y 2007); los cuales han permitido recuperar materiales arqueológicos (cerámica, artefactos líticos, de madera, textiles, metales, restos óseos humanos y animales, etc.); contribuyendo así a generar nuevos conocimientos sobre el sitio, filiación cultural, funcionalidad, cronología, etc.

También es característica de la gestión cultural el desarrollo de actividades referidas a la conservación. En los proyectos de Cajamarquilla se han realizado intervenciones en las estructuras de barro, por el deterioro en que se encontraban, así como en los materiales recuperados de las excavaciones (cerámica, óseo, textil, metal, etc.); es el caso de los trabajos arqueológicos y de conservación realizados por el Dr. Arturo Jiménez (1985) en el Conjunto Laberinto; Proyecto Arqueológico Cajamarquilla (PAC) (1996-2007), realizaron intervenciones en algunas estructuras de barro de Cajamarquilla; y las efectuadas por Walde (2002 y 2003) en la Calle Inca y en el Conjunto Sestieri, Héctor Walde (2002 y 2003) y Héctor Walde (2006 y 2007).

7. Libro Blanco de la gestión del Patrimonio Histórico-arqueológico del Grupo de Ciudades Patrimonio de la Humanidad de España/UNESCO. Ministerio de Educación, Cultura y Deporte (2015, p. 29).

6. Ministerio de Cultura. S/f. http://www.cultura.gob.pe/es/defensapatrimonio/participacionciudadana/cajamarquilla. Revisado el 21 de marzo del 2017. 
Los otros elementos que forman parte del proceso de gestión, son la protección y socialización o difusión como también se le denomina. Algunas actividades de este tipo han sido puestas en práctica en los diferentes proyectos de Cajamarquilla, como el Proyecto Arqueológico Cajamarquilla (PAC) (1996-2007): bajo la dirección de diferentes arqueólogos: Juan Mogrovejo (1996-1997), Dante Casareto (1998-1999), Héctor Walde (2002 y 2003) y Héctor Walde (2006 y 2007), en los cuales se busca involucrar la participación de la población del entorno inmediato al sitio de Cajamarquilla en los temas referidos a la difusión y protección.

Es destacable la labor de difusión y protección que se ha venido dando en el marco de los proyectos arqueológicos, plasmadas en los diferentes informes finales citados en este artículo. Se puede observar el trabajo inicial con la población aledaña respecto al sitio arqueológico a fin de proteger y conservar el sitio. Es necesario que la nueva información producto de la investigación, sea transmitida y compartida con la comunidad, ya que solo contando con el conocimiento social del patrimonio se puede pretender su conservación.

Finalmente otro elemento del proceso de gestión, en el sitio, es la interpretación ${ }^{8}$; el cual se puede dar bajo la forma del centro de interpretación"; es así que el Centro de Interpretación de Cajamarquilla, realiza la función de presentar el sitio, a través de las campañas de difusión y sensibilización de la población del entorno inmediato; actividades que buscan la protección del sitio.

\section{CONSIDERACIONES FINALES}

A partir de la evaluación de los informes finales de los proyectos realizados en el sitio, y a las actividades que se desarrollan en el sitio arqueológico de Cajamarquilla por el Centro de Interpretación, nos permite ver varios elementos del proceso de gestión, es así que se ha desarrollado: se ha avanzado en la investigación, obteniendo nuevos conocimientos sobre el sitio, cronología, filiación cultural, funcionalidad $\mathrm{y}$ de otros aspectos del sitio.

Por otro lado, se ha realizado trabajos de conservación en las estructuras de barro del sitio, entre las que destacan, el realizado por el Dr. Arturo Jiménez (1985), en el Conjunto Laberinto; y las efectuadas por Walde (2002 y 2003) en la Calle Inca y el Complejo Administrativo Ceremonial (Conjunto Sestieri) y los del Proyecto Arqueológico Cajamarquilla (PAC) (1996-2007); en dichos proyectos también se efectuaron la conservación de los materiales arqueológicos.

8. La “interpretación”, “presentación” o “puesta en valor”, muchos autores se refieren a lo mismo. (Hernández, 2002, p. 132). En el presente artículo al hablar de la presentación nos referirnos a la interpretación.

9. Ibid. La presentación [interpretación] puede darse bajo la forma de museos, centros de interpretación, parques culturales y/o naturales. 
El otro elemento presente es la difusión y protección que se ha venido dando en el marco de los proyectos arqueológicos. Se puede observar el trabajo inicial con la población aledaña a fin de proteger y conservar el sitio, algo muy importante considerando que el patrimonio es un legado, y sólo tiene valor en la medida en que es reconocido y alcanza un significado en la vida de la comunidad, fortaleciendo su identidad. (Monsalve, 2011, p. 39). Por ello, es necesario que la nueva información producto de la investigación, sea transmitida y compartida por el resto de la comunidad.

Consideramos que la difusión juega un rol importante en la protección del sitio, ya que permite conocerlo y, consecuentemente, protegerlo. Es necesario conocer el patrimonio para desear su conservación (Pérez, 2006, p. 11). Es lo que se buscó realizar en los proyectos arqueológicos, con sus programas de difusión; este es el mismo compromiso del Centro de Interpretación de Cajamarquilla, de crear conciencia en la población del entorno inmediato al sitio arqueológico de Cajamarquilla, a fin de protegerlo y preservarlo.

Por lo expuesto, en Cajamarquilla tenemos un proceso de gestión que en sus inicios fue discontinua, pero que con la implementación del Centro de Interpretación está adquiriendo mayor estabilidad.

Consideramos que en Cajamarquilla falta mucho por hacer urge labores investigación, protección y difusión para su preservación, se requiere una mayor actuación de las autoridades que por competencia le corresponden.

Otro tema de gran trascendencia es la promoción de la participación de los diferentes actores (Ministerio de Cultura, gobierno local, empresa privada y la población local), a fin de proteger, difundir y preservar el patrimonio arqueológico de este lugar, sin duda de importancia mayor. Asimismo, esta actuación en conjunto permite que los proyectos ejecutados desde esta perspectiva tengan sostenibilidad en el tiempo.

Hay una valiosa experiencia que se dio en Cajamarquilla, cuando se desarrolló el Proyecto Arqueológico Cajamarquilla 1996-2007, entre otros participaron la Refinería de Zinc de Cajamarquilla, la Fundación del Banco Wiese, Instituto Superior de Conservación YachayWasi. Por otra parte, la implementación del Centro de Interpretación de Cajamarquilla se realizó con el apoyo de la ONG Cesal, La Universidad Privada Cede Sapientae y la Agencia Española para la Cooperación Extranjera).

Asimismo, los proyectos arqueológicos que se realicen a futuro deberían tener el componente social, incorporar a la población aledaña que vive próximo al Sitio Arqueológico de Cajamarquilla, a fin de fomentar el conocimiento y valoración del patrimonio para de esta manera conseguir la conservación del sitio arqueológico.

Del mismo modo, solo creando conciencia del valor que tiene el patrimonio para los pobladores, ellos tendrán el deber de cuidar y el derecho de reclamar su conservación y otras actividades concernientes a su protección, difusión e investigación; 
por ello la valoración del patrimonio es un elemento importante de la gestión que debe considerarse en Cajamarquilla.

Finalmente, la información presentada muestra lo avanzado en la investigación del Sitio Arqueológico de Cajamarquilla queda aún mucho por estudiar y aprovechar del gran potencial arqueológico para investigar desde diferentes perspectivas siendo una de ellas, la gestión.

\section{REFERENCIAS BIBLIOGRÁFICAS}

Casareto, D. (1999). Proyecto Arqueológico Cajamarquilla. Informe Final. Temporada 1998. Refinería de Zinc de Cajamarquilla S.A -Fundación Augusto N. Wiesse.

Casareto, D. (2000). Proyecto Arqueológico Cajamarquilla. Informe Final. Temporada 1999. Refinería de Zinc de Cajamarquilla S.A -Fundación Augusto N. Wiesse.

Franco, R. (1998). Cajamarquilla. La Arquitectura de un Gran Centro Prehispánico de la Costa Central. Arkinka. Revista de arquitectura, diseño y construcción, 3(36), 72-89. Lima.

Ghezzi, I. (2007). Proyecto Arqueológico Cajamarquilla. Informe Final. Temporada 2004-2005.

Hernández, M. (2000). Patrimonio cultural y desarrollo sostenible en la quebrada de Humahuaca. Potencial y perspectivas. Cuadernos de la Facultad de Humanidades y Ciencias Sociales -Universidad Nacional de Jujuy, 18, pp. 125-152.

Ministerio de Cultura. (2015). ¿Qué es patrimonio cultural?. Ministerio de Cultura. Lima

Mariano, M., Endere, M., Mariano., C. (2014). Herramientas metodológicas para la gestión del patrimonio intangible. El caso del municipio de Olavarría, Buenos Aires, Argentina. Revista Colombiana de Antropología, 50, (2), 243- 269.

Menzel, D. (1968). La Cultura Huari. Las grandes civilizaciones del Perú Antiguo T. VI. Compañía de seguros y reaseguradoras Peruano - Suiza, 1968, Lima- Perú.

Mogrovejo, J. (1998). Proyecto Arqueológico Cajamarquilla. Conjunto Tello. Informe Final. Temporada 1997. Refinería de Zinc de Cajamarquilla S.A-Fundación Augusto N. Wiesse.

Mogrovejo, J. (1999). Cajamarquilla y el fin de la cultura Lima. En BIRA, 26, pp. 227243. Lima- Perú

Mogrovejo, J., Makowski., K. (1999). Cajamarquilla y los Mega Niños en el pasado prehispánico. Iconos, 1 (1), 46- 57

Monsalve, L. (2011). Gestión del Patrimonio Cultural y Cooperación Internacional. Cuadernos de Cooperación para el Desarrollo 6. Escuela Latinoamericana de Cooperación y Desarrollo. Medellín, Colombia. 
Narváez, J. (2004). Investigaciones Arqueológicas en Cajamarquilla. Excavaciones en el Sector XI del Conjunto Tello y la importancia de la ocupación Ichma en Cajamarquilla. Tesis para optar el título profesional de Licenciado en Arqueología. Universidad Nacional Mayor de San Marcos. Facultad de Ciencias Sociales. Lima-2004.

Narváez, J. (2006). Sociedades de la antigua ciudad de Cajamarquilla. Investigaciones arqueológicas en el sector XI del Conjunto Tello y un estudio de la colección tardía del Conjunto Sestieri. Avqi Ediciones. Lima-Perú, 2006.

Narváez, J. (s/f). Una Visión General del Desarrollo de las Sociedades Prehispánicas del Valle del Rímac. En: http://www.geocities.ws/contextos_peru/ctxt1-3. htm Revisado el 20.05.2017

Pérez, Amalia- Juez Gil. (2006). Gestión del Patrimonio Arqueológico. El yacimiento como recurso turístico. Editorial Ariel. 1a edición 2006. Barcelona-España.

Ruiz, C. (2013). "Patrimonio nacional arqueológico. El decomiso de bienes muebles de la época precolombina" en M. Aguilar, y O. Niglio (Ed.), La conservación del patrimonio cultural en Costa Rica. Esempi di architettura 15, pp. 163-176. https://dialnet.unirioja.es/servlet/articulo?codigo=5239055

Segura, R. (2001). Rito y Economía en Cajamarquilla. Investigaciones Arqueológicas en el Conjunto Arquitectónico Julio C. Tello. Fondo editorial de la Pontificia Universidad Católica del Perú. Primera Edición, 2001. Lima- Perú.

UNESCO, Ministerio de Educación, Cultura y Deporte. (2015). Libro Blanco de la gestión del Patrimonio Histórico-arqueológico del Grupo de Ciudades Patrimonio de la Humanidad de España. En: https://ciudadespatrimonio.org/publicaciones/1427495607_LIBROBLANCO.pdf

Walde, H. (2003). Proyecto Arqueológico Cajamarquilla. Informe Final. Temporada 2002. Refinería de Zinc de Cajamarquilla S.A-Instituto de Conservación y Restauración Yachay Wasi.

Walde, H.(2007). Proyecto Arqueológico Cajamarquilla. Informe Final. Temporada 20062007. Instituto de Conservación y Restauración Yachay Wasi.

Zúñiga, L., Pérez, R. (2013). Los recursos construidos de valor patrimonial en un modelo de gestión ambiental urbana. EURE, 39(117), 69-90

Zamora, F. (2002). La gestión del patrimonio cultural en España: presente y futuro. Portal Iberoamericano de Gestión Cultural, Boletín GC. www.gestioncultural. org (consultado el 20 de agosto de 2016). 


\section{SOBRE EL AUTOR}

\section{Benito Cáceres Ramos}

Licenciado en Arqueología por la Universidad Nacional Mayor de San Marcos. Con estudios de maestría en Gestión del Patrimonio Cultural entre el 2010 y el 2011, en la misma universidad.

El área de estudio de su interés es la arqueología de la Costa Central desde el Periodo Horizonte Medio hasta el Horizonte Tardío; así como la investigación en el ámbito de la arqueología histórica. Asimismo, desarrolla investigaciones en temas vinculados a la gestión del patrimonio arqueológico en general y específicamente en el Sitio Arqueológico de Cajamarquilla en el marco del desarrollo de la tesis. Actualmente labora en el Ministerio de Cultura. 